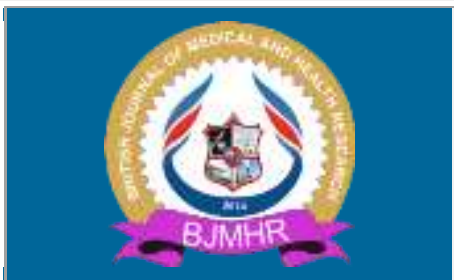

\title{
BJMHR
}

British Journal of Medical and Health Research

Journal home page: www.bjmhr.com

\section{Drug-Drug Interactions Mediated by P-Glycoprotein - An Overview}

\author{
Subhashri SHR, Vimalavathini R*, Kavimani S \\ College of Pharmacy, Mother Theresa Post Graduate and Research Institute of Health \\ sciences, Puducherry.
}

\begin{abstract}
Drug-drug interactions lead to lethal toxicities. Currently P-glycoprotein also plays significant role in drug-drug interactions. It is an efflux membrane transporter widely distributed throughout the body and is responsible for limiting the cellular uptake and the distribution of xenobiotics and toxic substance. The pharmacokinetics of a drug may be altered when co-administered with compounds which inhibit or induce P-glycoprotein pump which can affect pharmacodynamic and pharmacokinetic action of drugs. The present review summarizes various drug-drug interactions mediated by P-glycoprotein transporter.
\end{abstract}

Keywords: P-glycoprotein, drug efflux, drug interaction 


\section{INTRODUCTION}

Transporter mediated drug-drug interactions are being reported with increasing frequency ${ }^{1}$. The efflux transporter P-glycoprotein (P-gp) has received the most attention with regard to its role in restricting drug absorption and distribution and as a potential source for variability in drug pharmacokinetics and pharmacodynamics ${ }^{2}$. This transporter plays an important role in protecting sensitive tissues from toxic xenobiotics and can also interfere with the delivery of clinically used drugs.

P-gp belongs to the ATP-Binding Cassette (ABC) transporter family also known as MDR1 or ABCB1. It is expressed in various body tissues, such as liver, kidney, intestine, testis, and brain ${ }^{3}$. After drug administration, inhibition of P-gp by the drug can result in increased absorption of any co-administered drug that is P-gp substrate. Therefore, P-gp related interactions have critical clinical role and it is important to understand which drugs are substrates, inhibitors, or inducers of P-gp to minimize or avoid unwanted interactions ${ }^{1}$.

P-gp is highly expressed in cancer cells, leading to efflux of anticancer agents from cells leading to drug resistance. Moreover, the resistance to Central Nervous System (CNS) drugs, such as antidepressants and antiepileptic or anti-HIV medicine, also lead to P-gp over expression ${ }^{3}$. P-gp is one of the most recognized transport proteins that exhibit genetic polymorphism that might affect the outcome of drug therapy. Over 50 polymorphisms (single nucleotide polymorphisms and insertions/deletions) in the ABCB1 gene are known, and some of them appear to change the mRNA expression, protein expression and function of P-gp 4 . Co-administration of drugs that interact with P-gp as substrates, inhibitors or inducers can result in adverse drug-drug interactions. Induction or inhibition of these proteins also leads to drug interactions. Inhibition of this efflux transporter could increase the bioavailability of drugs. P-gp induction can, on the other hand, accelerate efflux transport and reduce the bioavailability of drugs ${ }^{5}$. List of drugs that interact with P-gp inhibitors and inducers are given in Table 1.

Drugs that are transported by P-gp are also metabolized by cytochrome P450 (CYP) isoform 3A4 (e.g., cyclosporine, antiepileptic drugs, antidepressant, fluoroquinolones, quinidine and ranitidine), which can confound interpretation of interactions ${ }^{6}$. P-gp may restrict the uptake of several antidepressants into the brain, thus limiting the distribution of these drugs into the brain and high rate of treatment failure ${ }^{7}$.

P-gp regulates the absorption and elimination of psychotropic drugs. Therefore, psychotropic drugs that are P-gp substrates may cause a drug interaction when P-gp inhibitors and inducers are co-administered, or when psychotropic drugs or other medicines those are P-gp substrates are added to a prescription ${ }^{8}$. This paper reviews some of the 
commonly used medicinal drugs which taken part in interaction of the drugs which is mediated by P-gp.

Table 1: Drug-drug interactions mediated by P-glycoprotein (MDR1)

\begin{tabular}{|c|c|c|c|}
\hline \multicolumn{4}{|c|}{ P-glycoprotein inhibitors } \\
\hline Sr.No & Drug & Inhibitor & Toxicity \\
\hline 1. & Digoxin & Quinidine & $\begin{array}{l}\text { Quinidine inhibit P-gp efflux, increase digoxin } \\
\text { bioavailability due to decreased renal clearance of } \\
\text { digoxin } 9\end{array}$ \\
\hline 2. & Digoxin & Atorvastatin & $\begin{array}{l}\text { Atorvastatin inhibit P-gp efflux, increase digoxin } \\
\text { bioavailability due to decreased renal clearance of } \\
\text { digoxin } 10\end{array}$ \\
\hline 3. & Digoxin & Verapamil & $\begin{array}{l}\text { Verapamil inhibit P-gp efflux, increase digoxin } \\
\text { bioavailability due to decreased renal tubular } \\
\text { elimination of digoxin }\end{array}$ \\
\hline 4. & Digoxin & Talinolol & $\begin{array}{l}\text { Talinolol inhibit P-gp efflux, increase digoxin } \\
\text { bioavailability and renal clearance of digoxin } \\
\text { remained unchanged }{ }^{13}\end{array}$ \\
\hline 5. & Digoxin & Clarithromycin & $\begin{array}{l}\text { Increase digoxin bioavailability by inhibiting P-gp } \\
\text { mediated tubular secretion of digoxin } 14\end{array}$ \\
\hline 6. & Digoxin & Itraconazole & $\begin{array}{l}\text { Itraconazole Inhibit P-gp efflux, increase digoxin } \\
\text { bioavailability due to decreased renal clearance of } \\
\text { digoxin } 15\end{array}$ \\
\hline 7. & Digoxin & Ritonavir & $\begin{array}{l}\text { Ritonavir inhibit P-gp efflux, increase digoxin } \\
\text { bioavailability by decreased renal clearance of } \\
\text { digoxin } 16\end{array}$ \\
\hline 8. & Digoxin & Omeprazole & $\begin{array}{l}\text { Omeprazole inhibit P-gp efflux, increase digoxin } \\
\text { bioavailability } 17\end{array}$ \\
\hline 9. & Digoxin & Cremophor EL & $\begin{array}{l}\text { Cremophor EL inhibit P-gp efflux, increase } \\
\text { digoxin bioavailability } 18\end{array}$ \\
\hline 10. & Paclitaxel & Cyclosporin & $\begin{array}{l}\text { Cyclosporin inhibit P-gp efflux, increase } \\
\text { paclitaxel bioavailability by decreased renal } \\
\text { clearance of paclitaxel lead to toxicity } 19\end{array}$ \\
\hline 11. & Paclitaxel & Elacridar $241 / 2$ & $\begin{array}{l}\text { Elacridar inhibit P-gp efflux, increase paclitaxel } \\
\text { bioavailability and lead to toxicity } 20\end{array}$ \\
\hline 12. & Paclitaxel & Valspodar & $\begin{array}{l}\text { Valspodar inhibit P-gp efflux, increase paclitaxel } \\
\text { bioavailability and lead to toxicity }\end{array}$ \\
\hline 13. & Docetaxel & Cyclosporine & $\begin{array}{l}\text { Cyclosporine inhibit P-gp efflux, increase } \\
\text { docetaxel bioavailability } 21,22\end{array}$ \\
\hline 14. & Saquinavir & Ritonavir & $\begin{array}{l}\text { Ritonavir inhibit P-gp efflux, increase saquinavir } \\
\text { bioavailability and lead to toxicity } 20\end{array}$ \\
\hline 15 . & Topotecan & Elacridar & $\begin{array}{l}\text { Elacridar inhibit P-gp efflux, increase topotecan } \\
\text { bioavailability and lead to toxicity } 20\end{array}$ \\
\hline 16. & Tacrolimus & Ketoconazole & $\begin{array}{l}\text { Increase tacrolimus bioavailability by inhibiting P- } \\
\text { gp efflux } 23\end{array}$ \\
\hline 17. & Tacrolimus & Clotrimazole & $\begin{array}{l}\text { Clotrimazole inhibit P-gp efflux, increase } \\
\text { Tacrolimus bioavailability } 24\end{array}$ \\
\hline 18. & Talinolol & Erythromycin & $\begin{array}{l}\text { Increase talinolol bioavailability due to increased } \\
\text { intestinal net absorption by inhibiting P-gp efflux } \\
\text { by erythromycin } 25\end{array}$ \\
\hline
\end{tabular}




\begin{tabular}{|c|c|c|c|}
\hline 19. & Talinolol & $\begin{array}{l}\text { D- } \alpha \text {-tocopheryl } \\
\text { polyethylene glycol } \\
1000 \text { succinate } \\
\text { (TPGS) }\end{array}$ & $\begin{array}{l}\text { TPGS inhibit P-gp efflux, increase talinolol } \\
\text { bioavailability } 26\end{array}$ \\
\hline 20. & Daunorubicin & Polysorbate 80 & $\begin{array}{l}\text { Polysorbate } 80 \text { inhibit P-gp efflux and increase } \\
\text { daunorubicin intracellular levels in cell cultures } 20\end{array}$ \\
\hline 21. & Saquinavir & Cremophor EL & $\begin{array}{l}\text { Cremophor EL increase saquinavir bioavailability } \\
\text { by inhibiting P-gp activity } 27\end{array}$ \\
\hline 22. & Loperamide & Quinidine & $\begin{array}{l}\text { Quinidine inhibit P-gp efflux, increase loperamide } \\
\text { entry in CNS causing Respiratory depression } 28\end{array}$ \\
\hline 23. & Sulfonylurea & $\begin{array}{l}\text { Clarithromycin, } \\
\text { Verapamil }\end{array}$ & $\begin{array}{l}\text { Inhibition of P-gp efflux, elevated risk of } \\
\text { hypoglycemia due to increased bioavailability of } \\
\text { sulfonylurea }{ }^{29}\end{array}$ \\
\hline 24. & Repaglinide & Atazanavir & $\begin{array}{l}\text { Atazanavir inhibit P-gp efflux, increase } \\
\text { repaglinide bioavailability and decreased renal } \\
\text { clearance of repaglinide }\end{array}$ \\
\hline 25. & Dabigatran & $\begin{array}{l}\text { Ketoconazole, } \\
\text { amiodarone, } \\
\text { verapamil,ticagrelor } \\
\text { and clarithromycin } \\
\end{array}$ & $\begin{array}{l}\text { Inhibit P-gp drug efflux, increase dabigatran } \\
\text { bioavailability and increased risk of severe } \\
\text { haemorrhage }\end{array}$ \\
\hline 26. & Methotrexate & $\begin{array}{l}\text { Omeprazole/ } \\
\text { pantoprazole }\end{array}$ & $\begin{array}{l}\text { Inhibit P-gp efflux, increase AUC and decrease } \\
\text { renal clearance } 20\end{array}$ \\
\hline 27. & Sorafenib & Verapamil & $\begin{array}{l}\text { Verapamil inhibit P-gp efflux, increase sorafenib } \\
\text { bioavailability by decrease renal clearance }\end{array}$ \\
\hline 28. & Erlotinib & Amiodarone & $\begin{array}{l}\text { Amiodarone inhibit P-gp efflux, increase erlotinib } \\
\text { bioavailability } 33\end{array}$ \\
\hline 29. & Fexofenadine & Lopinavir/ ritonavir & $\begin{array}{l}\text { Lopinavir/ ritonavir inhibit P-gp efflux, increase } \\
\text { fexofenadine bioavailability } 34\end{array}$ \\
\hline \multicolumn{4}{|c|}{ P-glycoprotein inducers } \\
\hline Sr.No & Drug & Inducer & Toxicity \\
\hline 1. & Digoxin & Rifampin & $\begin{array}{l}\text { Rifampin induce P-gp efflux, decrease digoxin } \\
\text { bioavailability due to increased renal clearance }\end{array}$ \\
\hline 2. & Talinolol & Rifampin & $\begin{array}{l}\text { Rifampin induce P-gp efflux, decrease talinolol } \\
\text { bioavailability } 36\end{array}$ \\
\hline 3. & Tacrolimus & Rifampin & $\begin{array}{l}\text { Rifampin induce P-gp efflux, decrease tacrolimus } \\
\text { bioavailability by increasing renal clearance of } \\
\text { tacrolimus }\end{array}$ \\
\hline 4. & Sulfonylurea & Rifampicin & $\begin{array}{l}\text { Rifampicin induce P-gp efflux, decrease } \\
\text { sulfonylurea bioavailability which lead to } \\
\text { therapeutic efficacy }{ }^{29}\end{array}$ \\
\hline 5. & $\begin{array}{l}\text { Dabigatran } \\
\text { etexilate }\end{array}$ & Rifampicin & $\begin{array}{l}\text { Rifampicin induce P-gp efflux, decrease } \\
\text { dabigatran etexilate bioavailability }{ }^{38}\end{array}$ \\
\hline 6. & Fexofenadine & Carbamazepine & $\begin{array}{l}\text { Carbamazepine induce P-gp efflux, decrease } \\
\text { fexofenadine bioavailability }\end{array}$ \\
\hline 7. & $\begin{array}{l}\text { Non-vitamin K } \\
\text { antagonist oral } \\
\text { anticoagulants } \\
\text { (NOACs) like } \\
\text { Dabigatran, } \\
\text { rivaroxaban, } \\
\text { apixaban, edoxaban }\end{array}$ & $\begin{array}{l}\text { Carbamazepine, } \\
\text { levetiracetam, } \\
\text { Phenobarbital, } \\
\text { phenytoin and } \\
\text { valproic acid }\end{array}$ & $\begin{array}{l}\text { Induce P-gp efflux thus decrease the effect of non- } \\
\text { vitamin K antagonist oral anticoagulants (NOACs) } \\
40\end{array}$ \\
\hline
\end{tabular}




\section{CONCLUSION}

P-gp is an efflux transporter pump present in many organs and plays an important role in drug transport. Expression of P-gp can have important effects on drug action. The importance of Pgp transporters in drug-drug interactions is increasingly being identified. The main causes of interactions are changes in the pharmacokinetics of drugs. Altered expression of this efflux pump can lead to lower therapeutic efficacy or greater toxicity. Knowledge of these potential drug interactions can help to ensure the provision of safety and effective treatment.

\section{REFERENCES}

1. Abbasi MM, Valizadeh H, Hamishehkar H, Zakeri-Milani P. Inhibition of Pglycoprotein expression and function by anti-diabetic drugs gliclazide, metformin, and pioglitazone in vitro and in situ. Research in Pharmaceutical Sciences.2016; 11(3): 177-186.

2. Hochman JH, Yamazaki M, Ohe T, Lin JH. Evaluation of Drug Interactions with PGlycoprotein in Drug Discovery: In Vitro Assessment of the Potential for Drug-Drug Interactions with P-Glycoprotein. Current Drug Metabolism. 2002; 3(3): 257 - 273.

3. Colabufo NA, Berardi F, Perrone MG, Capparelli E, Cantore M, Inglese C, Perrone R. Substrates, Inhibitors and Activators of P-glycoprotein: Candidates for Radiolabeling and Imaging Perspectives. Current Topics in Medicinal Chemistry.2010; 10:1703-1714.

4. Sharom FJ. The P-glycoprotein multidrug transporter. Biochemical Society Essays Biochem. 2011; 50:161-178.

5. Cascorbi I. Drug Interactions-Principles, Examples and Clinical Consequences. Dtsch Arztebl Int. 2012; 109(33-34): 546-56.

6. Palleria C, Paolo AD, Giofre C, Caglioti C, Leuzzi G, Siniscalchi A, Sarro GD, Gallelli L. Pharmacokinetic drug-drug interaction and their implication in clinical management. J Res Med Sci. 2013; 18(7): 601-610.

7. O’Brien FE, Dinan TG, Griffin BT, Cryan JF. Interactions between antidepressants and Pglycoprotein at the blood-brain barrier: clinical significance of in vitro and in vivo findings. British Journal of Pharmacology.2012; 165:289-312.

8. Akamine Y, Furukori Y, Ieiri I, Uno T. Psychotropic Drug-Drug Interactions Involving PGlycoprotein. CNS Drugs. 2012; 26 (11): 959-973.

9. Wessler JD, Grip LT, Medndell J, Giugliano RP. The P-Glycoprotein Transport System and Cardiovascular Drugs. Journal of the American College of Cardiology. 2013; 61(25):2495-502. 
10. Boyd RA, Stern RH, Stewart BH, Wu X, Reyner EL, Zegarac EA, Randinitis EJ, Whitfield L. Atorvastatin Coadministration May Increase Digoxin Concentrations by Inhibition of Intestinal P-Glycoprotein-Mediated Secretion. Clinical Pharmacology \& Therapeutics. 2000; 40(1):91-98.

11. Verschraagen M, Koks CHW, Schellens JHM, Hbeijnen J. P-Glycoprotein System As A Determinant Of Drug Interactions: The Case Of Digoxin-Verapamil. Pharmacological Research.1999; 40(4):301-306.

12. Ledwitch KV, Barnes BW, Roberts AG. Unravelling the complex drug-drug interactions of the cardiovascular drugs, verapamil and digoxin, with P-glycoprotein. Bioscience Reports.2016; 36:1-14.

13. Westphal K, Weinbrenner A, Giessmann T, Stuhr M, Franke G, Zschiesche M, Oertel R, Terhaag B, Kroemer HK, Siegmund W. Oral bioavailability of digoxin is enhanced by talinolol: Evidence for involvement of intestinal P-glycoprotein. Clinical Pharmacology \& Therapeutics.2000; 68:6-12.

14. Wakasugi H, Yano I, Ito T, Hashida T, Futami T, Nohara R, Sasayama S, Inui K. Effect of clarithromycin on renal excretion of digoxin: interaction with $\mathrm{P}$ glycoprotein. Clin Pharmacol Ther. 1998; 64(1):123-8.

15. Jalava, Maija K, Partanen, Juhani, Neuvonen, Pertti J. Itraconazole Decreases Renal Clearance of Digoxin.Therapeutic Drug Monitoring. 1997; 19 (6):609-613.

16. Phillips EJ, Rachlis AR, Itoa S. Digoxin toxicity and ritonavir: a drug interaction mediated through p-glycoprotein? AIDS.2003; 17:1577-1578.

17. Li W, Zeng S, Yu LS, Zhou Q. Pharmacokinetic drug interaction profile of omeprazole with adverse consequences and clinical risk management. Therapeutics and Clinical Risk Management. 2013; 9:259-271.

18. Tayrouz Y, Ding R, Burhenne J, Riedel KD, Weiss J, Tichy TH, Haefeli WE, Mikus G. Pharmacokinetic and Pharmaceutical interaction between digoxin and Cremophor RH40. Clinical Pharmacology \& Therapeutics.2003; 73:397-405.

19. Terwogt JMM, Beijnen JH, Huinink WHTB, Rosing H, Schellens JHM. Coadministration of cyclosporin enables oral therapy with paclitaxel. The Lancet.1998; 352(9124):285.

20. Marchetti S, Mazzanti R, Beijnen JH, Schellens JHM. Concise Review: Clinical Relevance of Drug-Drug and Herb-Drug Interactions Mediated by the $\mathrm{ABC}$ Transporter ABCB1 (MDR1, Pglycoprotein). The Oncologist. 2007; 12:927-941. 
21. Malingré MM, Richel DJ, Beijnen JH, Rosing H, Koopman FJ, Huinink WWTB. Coadministration of Cyclosporine Strongly Enhances the Oral Bioavailability of Docetaxel. J Clin Oncol. 2001; 19(4):1160-6.

22. Chiou WL, Wu TC, Ma C, Jeong HY, Malingré MM, Beijnen JH ,Schellens JHM. Enhanced Oral Bioavailability of Docetaxel by Coadministration of Cyclosporine: Quantitation and Role of P-Glycoprotein. Journal of Clinical Oncology.2002;20(7):1951-1957.

23. Hebert MF. Contributions of hepatic and intestinal metabolism and P-glycoprotein to cyclosporine and tacrolimus oral drug delivery. Advanced Drug Delivery Reviews. 1997; 27:201-214.

24. Choy M. Tacrolimus Interaction with Clotrimazole. P\&T. 2010; 35(10):568-569.

25. Schwarz UI, Gramatte T, Krappweis J, Oertel R, Kirch W. P-glycoprotein inhibitor erythromycin increases oral bioavailability of talinolol in humans. International Journal of Clinical Pharmacology and Therapeutics.2000; 38(4):161-167.

26. Bogman K, Zysset Y, Degen L, Hopfgartner G, Gutmann H, Alsenz J, Drewe J. PGlycoprotein and Surfactants: Effect on Intestinal Talinolol Absorption. Clinical Pharmacology \& Therapeutics.2005; 77:24-32.

27. Martin-Facklam M, Burhenne J, Ding R, Fricker R, Mikus G, Walter-Sack I, Haefeli WE. Dose-dependent increase of saquinavir bioavailability by the pharmaceutics aid cremophor EL. Br J Clin Pharmacol. 2002; 53: 576-581.

28. Sadeque AJ, Wandel C, He H, Shah S, Wood AJ. Increased drug delivery to the brain by Pglycoprotein inhibition. Clin Pharmacol Ther. 2000;68(3):231-7.

29. May M, Schindler C. Clinically and pharmacologically relevant interactions of antidiabetic drugs. Ther Adv Endocrinol Metab.2016; 7(2): 69-83.

30. Goud T, Maddi S, Devanna N, Prasad TR. Evaluation of pharmacokinetic and pharmacodynamic interaction between repaglinide and atazanavir in healthy, diabetic and hepatic impaired rats: possible inhibition of CYP3A, OATP, and P-glycoprotein transporters. ADMET \& DMPK .2016; 4(3):269-279.

31. Finch A, Pillans P. P-glycoprotein and its role in drug-drug interactions. Aust Prescr. 2014; 37:137-9.

32. Wang X, Zhang X,Huang X, Li Y, Wu M, Liu J. The drug-drug interaction of sorafenib mediated by P-glycoprotein and CYP3A4. Xenobiotica. 2015; 46: 651-658.

33. Lopez Brunso M, Toro Blanch C, Sais Girona E, Roa Garcia D, Hernández Martinez A, Izquierdo Font A, Guerra Prio S, Mas Pueyo HG, Bosch-Barrera J.Probable drug- 
drug interaction between erlotinib and amiodarone causes severe neurotoxicity in a patient with advanced lung cancer.Anti-Cancer Drugs. 2018; 29(4):380-383.

34. Van Heeswijk RPG, Bourbeau M, Campbell P, Seguin I, Bobby M. Chauhan, Brian C. Foster. Time-dependent interaction between lopinavir/ritonavir and fexofenadine. Journal of Clinical Pharmacology. 2006; 46(7):758.

35. Greiner B, Eichelbaum M, Fritz P, Kreichgauer PH, Richter OV,Zundler J, Kroemer HK. The role of intestinal P-glycoprotein in the interaction of digoxin and rifampin.The Journal of Clinical Investigation.1999; 104(2):147-153.

36. Westphal K, Weinbrenner A, Zschiesche M, Franke G, Knoke M, Oertel R, Fritz P, Richter OV, Warzok R, Hachenberg T, Kauffmann HM, Schrenk DS, Terhaag B, Kroemer HK, Siegmund W. Induction of P-glycoprotein by rifampin increases intestinal secretion of talinolol in human beings: A new type of drug/drug interaction. Clinical Pharmacology \& Therapeutics.2000; 68:345-355.

37. Hebert MF, Fisher RM, Marsh CL, Dressler D, Bekersky I. Effects of rifampin on tacrolimus pharmacokinetics in healthy volunteers. J Clin Pharmacol. 1999; 39(1):916.

38. Hartter S, Koenen-Bergmann M, Sharma A, Nehmiz G, Lemke U, Timmer W, Reilly PA. Decrease in the oral bioavailability of dabigatran etexilate after co-medication with rifampicin. Br J Clin Pharmacol. 2012; 74(3): 490-500.

39. Yamada S, Yasui-Furukori N, Akamine Y, Kaneko S, Uno T. Effects of the Pglycoprotein inducer carbamazepine on fexofenadine pharmacokinetics. Ther Drug Monit. 2009; 6:764-8.

40. Stollberger C, Finsterer J. Interactions between non-vitamin K oral anticoagulants and antiepileptic drugs. 26. Epilepsy Research.2016; 126:98-101.

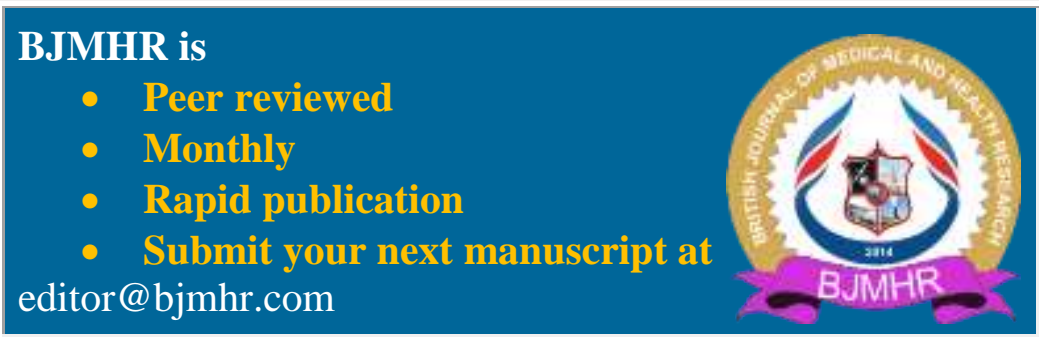

